\title{
Non-invasive assessment by Doppler ultrasound of 155 patients with bioprosthetic valves: a comparison of the Wessex porcine, low profile Ionescu-Shiley, and Hancock pericardial bioprostheses
}

\author{
I A SIMPSON, I J REECE, A B HOUSTON, I HUTTON, D J WHEATLEY, \\ $S$ M COBBE
}

From the University Departments of Medical Cardiology and Cardiac Surgery, Royal Infirmary, Glasgow

SUMMARY One hundred and fifty five patients with 167 bioprosthetic valves (68 Wessex porcine, 54 Hancock pericardial, and 45 low profile Ionescu-Shiley pericardial valves) were studied by Doppler ultrasound. Valve gradients were calculated from the mitral and aortic flow velocities by the modified Bernoulli equation. Mean mitral gradients were significantly smaller across the Ionescu-Shiley valves than across the Wessex porcine or Hancock pericardial valves. Mitral pressure half time was, however, significantly longer in the Hancock pericardial than in the Wessex porcine or Ionescu-Shiley valves. No significant differences were seen among the groups of aortic bioprostheses, though the comparable size of Wessex porcine valves showed significantly higher gradients. Bioprosthetic regurgitation was detected in 13 of 103 mitral and 11 of 59 aortic valves, though it was suspected clinically in only 12 mitral and six aortic bioprostheses.

Doppler ultrasound is a repeatable non-invasive method of acquiring haemodynamic information in vivo from a variety of bioprostheses and it can detect bioprosthetic regurgitation at an early stage.

There is considerable interest in the haemodynamic assessment of the newer bioprostheses. Although some in vitro haemodynamic information is available, ${ }^{1}$ this is not easily extrapolated to the clinical situation. In addition the in vivo assessment of valve prostheses has usually been obtained intraoperatively ${ }^{23}$ and rarely at cardiac catheterisation, ${ }^{4}$ and there are no in vivo haemodynamic data on the bioprostheses that we studied. Noninvasive haemodynamic measurements are important in the continuing clinical assessment of new bioprostheses because the alternative is repeated invasive investigation, which has major ethical implications in otherwise healthy individuals. In addition, in patients in whom important prosthetic dysfunction is suspected, cardiac catheterisation is

Requests for reprints to Dr I A Simpson, University Department of Medical Cardiology, Royal Infirmary, 10 Alexandra Parade, Glasgow G31 2ER.

Accepted for publication 3 March 1986 nearly always required to evaluate its nature and severity. A non-invasive method of accurately assessing prosthetic function in vivo would be valuable. Echocardiography can show excellent structural detail but is an insensitive method for detecting prosthetic dysfunction and does not provide haemodynamic information. 56

Doppler ultrasound predicts valve gradients in patients with mitral ${ }^{7-9}$ and aortic ${ }^{10-12}$ stenosis and can identify valve regurgitation in these patients. ${ }^{13-15}$ More recently these techniques have been applied to the assessment of valve gradients in a small number of patients with either mechanical ${ }^{16-18}$ or bioprosthetic ${ }^{18-20}$ valve replacements. Doppler ultrasound was also of value in the assessment of prosthetic valve regurgitation in a larger series of mechanical and bioprosthetic valves. ${ }^{21}$ We have shown the validity of Doppler ultrasound measurement of valve gradients across bioprosthetic valves in vitro ${ }^{22}$ and have investigated the accuracy of assessing mitral prosthetic gradients 
and the presence and extent of mitral regurgitation by Doppler ultrasound in vivo. ${ }^{23}$ The purpose of this study was to determine the value of Doppler ultrasound in the haemodynamic assessment of new types of bioprosthetic valve replacements.

\section{Patients and methods}

We studied 155 patients (age range 33 to 76 years, mean 55.8 years) with 167 bioprostheses. This group comprised 68 with Wessex porcine bioprostheses (47 mitral, 21 aortic), 54 with Hancock pericardial bioprostheses (27 mitral, 27 aortic), and 45 with low profile Ionescu-Shiley pericardial valves $(29 \mathrm{mitral}$, 16 aortic). Valve sizes ranged from $29 \mathrm{~mm}$ to $35 \mathrm{~mm}$ for the mitral bioprostheses and from $19 \mathrm{~mm}$ to $27 \mathrm{~mm}$ for aortic bioprostheses.

Before Doppler ultrasound examination all patients underwent cross sectional echocardiography with a Diasonics V3400R phased array echocardiographic imaging system. Measurements were made of left ventricular, right ventricular, and left atrial dimensions, and left ventricular wall thickness. Prosthetic valve function was assessed visually by leaflet separation and apposition and by the structure and motion of the valve ring. Doppler examination was performed by means of a Vingmed Alfred pulsed and continuous wave Doppler velocimeter with a $2 \mathrm{MHz}$ transducer that was interfaced with a Doptek spectrum analyser. Doppler examination was performed with the patient at rest immediately after echocardiographic examination. The optimal Doppler signal was obtained by transducer manipulation and was based both on the audiosignal and on the visual display of a clearly demarcated spectral envelope on the spectrum analyser. Patients with a mitral bioprosthesis who were in atrial fibrillation had analysis performed on Doppler signals obtained during similar diastolic time periods.

Mitral flow was detected from the apical position and the peak mitral diastolic flow velocity was measured in the continuous wave mode. We calculated the transprosthetic valve gradient by means of the modified Bernoulli equation ${ }^{8}: P=4 V^{2}$, where $P$ is the valve gradient in $\mathrm{mm} \mathrm{Hg}$ and $\mathrm{V}$ is the maximum velocity in $\mathrm{m} / \mathrm{s}$.

This equation was applied to the maximum mitral diastolic flow velocity at each $10 \mathrm{~ms}$ interval throughout the diastolic time period and these results were averaged to obtain the mean mitral valve gradient. In addition we measured the mitral pressure half time, that is the time taken for the mitral diastolic flow velocity to fall to the equivalent of half the initial calculated peak pressure drop. In mitral stenosis this provided an assessment of the degree of mitral obstruction that was independent of cardiac output $^{24}$ and it has also been used to assess a small number of mitral valve prostheses. ${ }^{16}$

We used continuous wave Doppler from the apical position to assess mitral regurgitation, which was indicated by high velocity systolic flow away from the transducer into the left atrial cavity. The pulsed wave mode, which allowed depth resolution of the Doppler signal, was then used to estimate the degree of mitral regurgitation, as previously reported, ${ }^{23}$ by measurement of the extent of flow into the left atrial cavity. Although it is not possible to provide precise quantification of the degree of mitral prosthetic regurgitation by this method it does allow identification of those patients with severe regurgitant lesions.

We measured aortic prosthetic flow velocity in the continuous wave mode from various precordial positions that have been used to assess aortic stenosis $^{12}$ - suprasternal notch, apex, subcostal, supraclavicular, and right parasternal in the right lateral decubitus position. The maximum systolic velocity with a clearly demarcated spectral signal was used to calculate the peak aortic valve gradient by the modified Bernoulli equation. The presence of aortic regurgitation was also determined from these precordial positions.

Tricuspid regurgitation was assessed from either the apical or left parasternal positions and when present it was used to estimate right ventricular systolic pressure derived from the gradient obtained across the tricuspid valve during systole. Assuming the absence of pulmonary valve disease the pulmonary artery systolic pressure could be determined. ${ }^{25}$

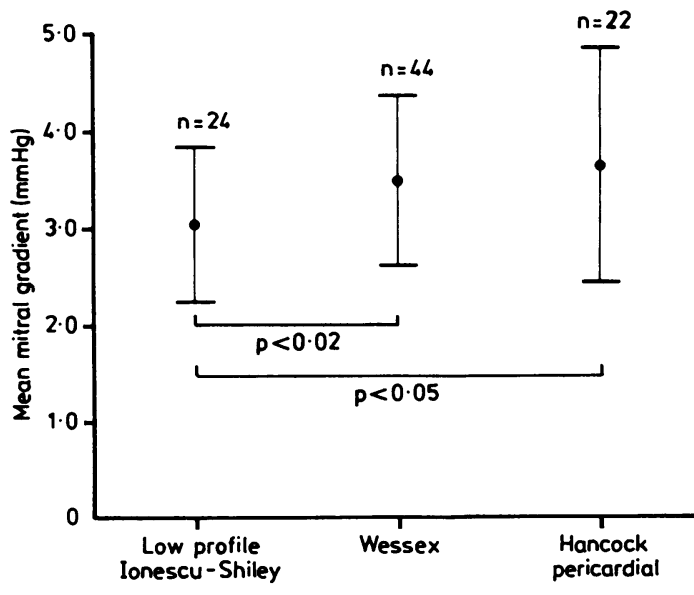

Fig. 1 Comparison of mean mitral gradients (mean (SD) ) across all competent mitral bioprostheses. 


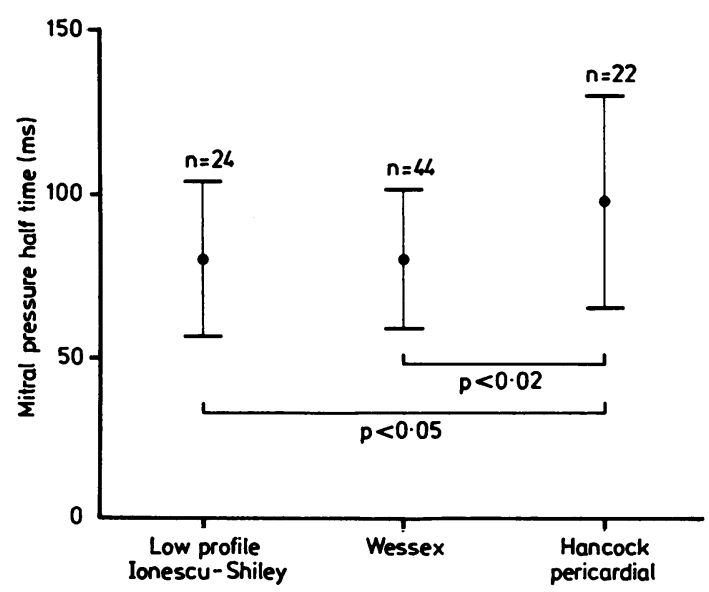

Fig. 2 Comparison of the mitral pressure half time (mean (SD) ) for all competent mitral bioprostheses.

STATISTICAL METHODS

Mean and standard deviations were calculated, and statistical analysis was performed by the unpaired $t$ test.

\section{Results}

We obtained satisfactory cross sectional echocardiograms in $133(86 \%)$ patients but these did not show any structural abnormality suggestive of prosthetic valve dysfunction. Doppler examination gave satisfactory recordings in all 103 mitral prostheses and in $59(92 \%)$ of the 64 aortic prostheses.

There was no significant difference in the resting heart rates between the three groups of patients (Wessex porcine 84 (7), Hancock pericardial 78(5), low profile Ionescu-Shiley 82 (9) beats/min).

\section{BIOPROSTHETIC GRADIENTS}

Figure 1 shows the mean mitral valve gradients measured by Doppler ultrasound. The presence of important mitral regurgitation will increase the mitral diastolic flow velocity and hence the measured valve gradient. Although this would produce an increased gradient even at catheterisation, to obtain a direct comparison between the three types of valve prostheses we excluded those valves demonstrating mitral regurgitation on the Doppler examination. There was a narrow range of valve gradients for each type of bioprosthesis despite the variation in valve size, and significantly lower gradients were found with the Ionescu-Shiley low profile valve than with either the Wessex porcine bioprosthesis $(p<0.02)$ or the Hancock pericardial valve $(p<0.05)$. Mitral pressure half time (Fig. 2) was, however, significantly longer with the Hancock pericardial bioprosthesis than with either the Wessex porcine bioprosthesis $(p<0.02)$ or the low profile IonescuShiley valve $(p<0.05)$; there was no significant difference in pressure half time between the Wessex porcine and low profile Ionescu-Shiley valves.

There was no significant difference in the peak aortic gradients across the three types of bioprostheses (Fig. 3).

Table 1 shows a comparison of all the mitral prostheses of various sizes. Only two patients had a $35 \mathrm{~mm}$ mitral bioprosthesis (both Wessex porcine valves) and they were excluded. The $29 \mathrm{~mm}$ mitral bioprosthesis had a significantly longer pressure half time than either the $31 \mathrm{~mm}$ or $33 \mathrm{~mm}$ sizes. There was no difference, however, in either the peak mitral flow velocity or mean mitral valve gradient among the three valve sizes. Table 2 compares the results for the three different bioprostheses of the same size. A significantly higher peak mitral flow velocity was demonstrated with the $29 \mathrm{~mm}$ Wessex porcine valve than with the other two bioprostheses, though this difference was not found with the $31 \mathrm{~mm}$ valves. For aortic bioprostheses with a diameter of $23 \mathrm{~mm}$ a significantly $(p<0.005)$ higher gradient was demonstrated across the Wessex porcine valve $(21 \cdot 4(9 \cdot 8)$ $\mathrm{mmHg}, \mathrm{n}=8$ ) than across the pericardial bioprostheses (Hancock pericardial $15 \cdot 1(5 \cdot 6) \mathrm{mm} \mathrm{Hg}$, $n=13$ and low profile Ionescu-Shiley $15 \cdot 6(4 \cdot 4)$ $\mathrm{mm} \mathrm{Hg}, \mathrm{n}=7$ ).

\section{BIOPROSTHETIC REGURGITATION}

Mitral regurgitation was detected in $13(12.6 \%)$ of 103 mitral bioprostheses: three of 47 Wessex porcine, five of 29 low profile Ionescu-Shiley, and five of 27 Hancock pericardial prostheses. It had been sus-

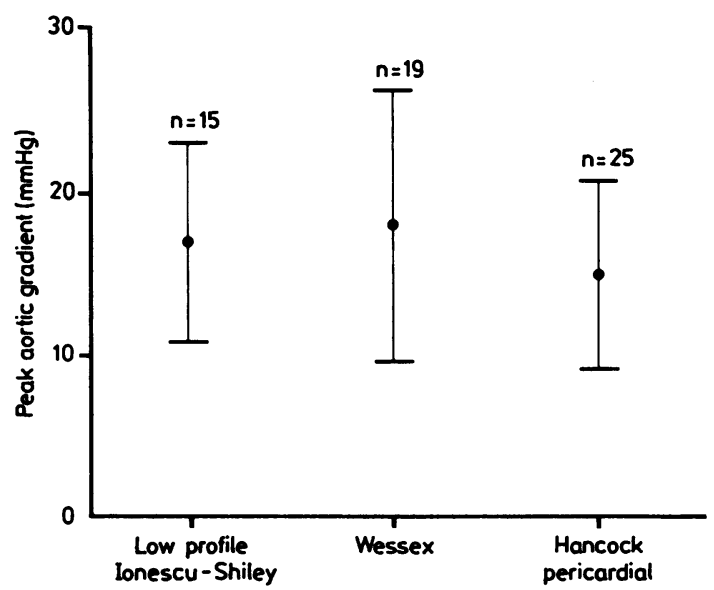

Fig. 3 Comparison of peak valve gradients (mean (SD)) across all competent aortic bioprostheses. 
Table 1 Haemodynamic variables (mean (SD)) and valve sizes for all mitral bioprostheses

\begin{tabular}{|c|c|c|c|}
\hline & \multicolumn{3}{|c|}{ Valve size (mm) } \\
\hline & $29(n=36)$ & $31(n=40)$ & $33(n=13)$ \\
\hline $\begin{array}{l}\text { Peak mitral flow velocity }(\mathrm{cm} / \mathrm{s}) \\
\text { Mean mitral gradient }(\mathrm{mm} \mathrm{Hg}) \\
\text { Mitral pressure half time (ms) }\end{array}$ & $\begin{array}{c}145.7(22.7) \\
3.52(1.07) \\
90.6(32)\end{array}$ & $\begin{array}{c}141 \cdot 1(23.4) \\
3.22(0.82) \\
80.0(20.0)^{\star}\end{array}$ & $\begin{array}{c}146.5(26 \cdot 2) \\
3.51(1 \cdot 38) \\
74.6(19 \cdot 0)^{\star \star}\end{array}$ \\
\hline
\end{tabular}

Table 2 Haemodynamic variables (mean (SD)) for bioprostheses of same size (29 mm and $31 \mathrm{~mm}$ )

\begin{tabular}{|c|c|c|c|c|c|c|}
\hline & \multicolumn{3}{|l|}{$29 \mathrm{~mm}$} & \multicolumn{3}{|l|}{$31 \mathrm{~mm}$} \\
\hline & $\begin{array}{l}\text { Wessex } \\
(n=9)\end{array}$ & $\begin{array}{l}\text { Hancock } \\
\text { pericardial } \\
(n=14)\end{array}$ & $\begin{array}{l}\text { Low profile } \\
\text { Ionescu-Shiley } \\
(n=13)\end{array}$ & $\begin{array}{l}\text { Wessex } \\
(n=22)\end{array}$ & $\begin{array}{l}\text { Hancock } \\
\text { pericardial } \\
(n=8)\end{array}$ & $\begin{array}{l}\text { Low profile } \\
\text { Ionescu-Shiley } \\
(n=10)\end{array}$ \\
\hline $\begin{array}{l}\text { Peak mitral flow } \\
\text { velocity (cm/s) } \\
\text { Mean mitral gradient } \\
\text { (mm Hg) } \\
\text { Mitral pressure half } \\
\text { time (ms) }\end{array}$ & $\begin{array}{c}166.4(17 \cdot 1) \\
3.69(0.61) \\
83.3(19.4)\end{array}$ & $\begin{array}{c}141.7(14.4)^{\star \star \star} \\
2.61(1.39) \\
105(36 \cdot 3)^{\star}\end{array}$ & $\begin{array}{c}135.7(25.3) \star \star \star \\
3.31(0.96) \\
80(30.3)\end{array}$ & $\begin{array}{c}141 \cdot 1(24.9) \\
3.31(0.83) \\
80(21.4)\end{array}$ & $\begin{array}{c}151.0(26.9) \\
3.57(1.02) \\
81.2(23.0)\end{array}$ & $\begin{array}{l}133.2(14.2) \\
2.74(0.37)^{\star \star} \\
79(15.2)\end{array}$ \\
\hline
\end{tabular}

${ }_{\star \star \star \star} p<0.005$ compared with $29 \mathrm{~mm}$ Wessex; ${ }^{\star \star} \mathrm{p}<0.01$ compared with both $31 \mathrm{~mm}$ Wessex and Hancock pericardial; ${ }^{\star} p<0.05$ compared with both $29 \mathrm{~mm}$ Wessex and low profile lonescu-Shiley.

pected clinically in 12 of the 13 patients. Table 3 shows the severity of mitral regurgitation graded by the extent of left atrial systolic flow. Three patients were graded as having severe mitral regurgitation, and all subsequently required operation. Additional evidence of severe bioprosthetic regurgitation was obtained in the two patients with a low profile Ionescu-Shiley bioprosthesis who, in the presence of a normal pressure half time, had peak mitral diastolic flow velocities of 240 and $232 \mathrm{~cm} / \mathrm{s}$, which is well outside the range for the competent prostheses (Table 4). The peak mitral velocity in the remaining patient with severe mitral regurgitation (in a Hancock pericardial bioprosthesis) and the peak mitral velocities associated with mild or moderate regurgitation all fell within the range for the competent valve prostheses.

Aortic regurgitation was detected in $11(18.6 \%)$ of 59 bioprostheses (five of 19 Wessex porcine, three of 15 low profile Ionescu-Shiley, and three of 25 Hancock pericardial), though this was suspected clinically in only six of the 11 patients.

Tricuspid regurgitation was detected by Doppler ultrasound in $30(29 \%)$ of the 103 mitral bioprostheses but was not found in any patient with single aortic valve replacement. Although the presence of tricuspid regurgitation was identified in $\mathbf{3 0}$ patients, a clearly demarcated spectral signal from which the peak velocity could be measured was obtained in only 24 of the 30 patients. The estimated pulmonary systolic pressure ranged from 15 to 33 $\mathrm{mm} \mathrm{Hg}$ (mean $21.9 \mathrm{~mm} \mathrm{Hg}$ ) in the 21 patients with a competent bioprosthesis, and was $22 \mathrm{~mm} \mathrm{Hg}$, $33 \mathrm{~mm} \mathrm{Hg}$, and $57 \mathrm{~mm} \mathrm{Hg}$ in the remaining three patients with mitral regurgitation.

\section{Discussion}

Doppler ultrasound has great potential as a noninvasive method of assessing bioprosthetic valve function. Although in vitro studies can provide data on the haemodynamic profile of various bioprostheses it is difficult to extrapolate from these results to the clinical situation.

The newer pericardial bioprostheses presented

Table 3 Severity of mitral regurgitation graded by extent of left atrial systolic flow

\begin{tabular}{llll}
\hline & Mild & Moderate & Severe \\
\hline $\begin{array}{l}\text { Wessex }(n=3) \\
\text { Low profile Ionescu-Shiley }\end{array}$ & 3 & - & - \\
$(\mathrm{n}=5)$ & 3 & - & 2 \\
Hancock pericardial $(\mathrm{n}=5)$ & 1 & 3 & 1 \\
\hline
\end{tabular}

Table 4 Peak mitral diastolic flow velocities $(\mathrm{cm} / \mathrm{s})$ for the competent bioprostheses

\begin{tabular}{lrl}
\hline & Range & Mean \\
\hline Wessex $(n=44)$ & $92-200$ & 149 \\
Low profile Ionescu-Shiley $(n=24)$ & $88-180$ & 134 \\
Hancock pericardial $(n=22)$ & $116-204$ & 143 \\
\hline
\end{tabular}


less obstruction to flow than the porcine bioprostheses when they were studied in vitro ${ }^{22}$ and intraoperatively, ${ }^{3}$ though it is not certain whether this is of clinical relevance.

The range of mean valve gradients for bioprostheses in the mitral position was quite narrow and gradients in all competent prostheses were acceptably low. We expected the valve gradients in the low profile Ionescu-Shiley pericardial valve to be lower than in the Wessex porcine bioprosthesis, but we did not expect them to be significantly lower than in the Hancock pericardial valve. Similarly the longer mitral pressure half time in the Hancock pericardial prosthesis suggests that the in vivo obstructive characteristics of this valve differ from those that were established in vitro.

The mean mitral valve gradient was significantly lower for the low profile Ionescu-Shiley valve only at the $31 \mathrm{~mm}$ size and not at the $29 \mathrm{~mm}$ size. The prolonged pressure half time noted with the Hancock pericardial valve, however, occurred only at $29 \mathrm{~mm}$ and not at $31 \mathrm{~mm}$. Because the pressure half time relates more to the initial phase of diastole and the mean mitral gradient relates to the whole diastolic time period, the difference in pressure half time noted with the Hancock pericardial valve could be explained by an increased restriction to flow during the first part of diastole, possibly as a result of less rapid leaflet opening, which may only be of relevance at the smaller valve size.

Similarly the low profile Ionescu-Shiley pericardial valve may be less obstructive throughout diastole and hence produce a significantly lower mean valve gradient with the $31 \mathrm{~mm}$ valve than Wessex or Hancock valves of similar size. This effect was also seen with the $29 \mathrm{~mm}$ size valves, but it was not significant. In vitro studies had not led us to expect these differences in mean valve gradient and mitral pressure half time, and such discrepancies indicate the complexity of the pressure flow relation across the mitral valve in vivo.

The slight reduction in the mitral pressure half time with the larger bioprostheses indicates a tendency to increased obstruction with smaller bioprostheses. There was no difference in the mean mitral valve gradients, however, which suggests that this may only have an effect at rest in the early diastolic period when valve flow is greater.

Although there was no overall difference between the three groups of aortic bioprostheses, the Wessex porcine valve, as expected, produced significantly higher peak aortic gradients than different valve types of similar size.

The ability of Doppler ultrasound to identify bioprosthetic valvar regurgitation may be valuable in the assessment of prosthetic valve dysfunction. In this study not only did we identify three patients with severe mitral regurgitation who required reoperation, but we detected mitral bioprosthetic regurgitation in 10 further patients who were symptom free. Although difficulties arise when pulsed Doppler is used to quantitate mitral regurgitation from the extent of left atrial systolic flow, the technique can allow identification of those with severe regurgitation, particularly where mitral diastolic flow velocity is also increased. Valvar regurgitation in patients with bioprostheses can be the result of leaflet tears, and by detection of regurgitation, Doppler ultrasound may identify at risk patients at an early stage. It is difficult to distinguish between a periprosthetic and a through-valve leak by Doppler ultrasound alone, and simultaneous cross sectional echocardiographic imaging, or more particularly real time colour coded Doppler, ${ }^{26}$ may improve performance. The question whether patients suspected of having less important mitral regurgitation will subsequently develop major leaflet tears will only be resolved by careful patient follow up and examination at reoperation.

Aortic regurgitation was identified in 11 aortic bioprostheses, one of which had to be replaced because of leaflet tear. Although it is not possible to measure aortic regurgitation accurately by Doppler ultrasound, its identification in conjunction with clinical and other non-invasive assessment may predict the development of important prosthetic dysfunction at an early stage.

The estimation of right ventricular and therefore pulmonary artery systolic pressure in patients with tricuspid regurgitation is a useful adjunct to the assessment of bioprosthetic valve function. This was possible in several patients with mitral bioprostheses who had tricuspid regurgitation, presumably as a result of right ventricular dilatation associated with previous mitral valve disease. In all patients with a competent mitral bioprosthesis pulmonary pressures were satisfactory at rest, though they were significantly increased in a patient with mitral regurgitation.

This study has demonstrated the successful application of Doppler ultrasound techniques in a large series of patients with bioprosthetic valves. It allowed a comparison of the haemodynamic function of different bioprosthetic valves in vivo. Because it is both non-invasive and can be repeated it is likely to have a major impact on the continuing assessment of the newer types of bioprostheses. The ability to identify the presence of bioprosthetic regurgitation in these patients and to provide information on its severity in patients with mitral bioprostheses suggests that non-invasive assessment of developing bioprosthetic dysfunction may be possible. 


\section{References}

1 Walker DK, Scotten LN, Brownlee RT. New generation of tissue valves - their in vitro function in the mitral position. $\mathcal{F}$ Thorac Cardiovasc Surg 1984; 88: 573-81.

2 Becker RM, Strom J, Frishman W, et al. Hemodynamic performance of the Ionescu-Shiley valve prosthesis. $f$ Thorac Cardiovasc Surg 1980; 80: 613-20.

3 Cosgrove DM, Lytle BW, Gill CC, et al. In vivo hemodynamic comparison of porcine and pericardial valves. f Thorac Cardiovasc Surg 1985; 89: 358-68.

4 Horstkotte D, Haerten K, Seipel L, et al. Central hemodynamics at rest and during exercise after mitral valve replacement with different prostheses. Circulation 1983; 68 (suppl II): II-161-8.

5 Effron MK, Popp RL. Two-dimensional echocardiographic assessment of valve dysfunction and infective endocarditis. I Am Coll Cardiol 1983; 2: 597-606.

6 Alam M, Lakier JB, Pickard SD, Goldstein S. Echocardiographic evaluation of porcine bioprosthetic valves: experience with 309 normal and 59 dysfunctioning valves. Am $\mathcal{f}$ Cardiol 1983; 52: 309-15.

7 Holen J, Aaslid R, Landmark K, Simonsen S. Determination of pressure gradient in mitral stenosis with a noninvasive ultrasound Doppler technique. Acta Med Scand 1976; 199: 455-60.

8 Hatle L, Brubakk A, Tromsdal A, Angelson B. Noninvasive assessment of pressure drop in mitral stenosis by Doppler ultrasound. Br Heart $\mathcal{f} 1978$; 40: 131-40.

9 Holen J, Simonsen S. Determination of pressure gradient in mitral stenosis with Doppler echocardiography. Br Heart f 1979; 41: 529-35.

10 Hatle L, Angelsen BA, Tromsdal A. Non-invasive assessment of aortic stenosis by Doppler ultrasound. $\mathrm{Br}$ Heart $\mathcal{f}$ 1980; 43: 284-92.

11 Berger M, Berdoff RL, Gallerstein PE, Goldberg E. Evaluation of aortic stenosis by continuous wave Doppler ultrasound. $f \mathrm{Am}$ Coll Cardiol 1984; 3: 150-6.

12 Simpson IA, Houston AB, Sheldon CD, Hutton I, Lawrie TDV. Clinical value of Doppler echocardiography in the assessment of adults with aortic stenosis. Br Heart $\mathcal{F}$ 1985; 53: 636-9.

13 Blanchard D, Diebold B, Peronneau P, et al. Noninvasive diagnosis of mitral regurgitation by Doppler echocardiography. Br Heart f 1981; 45: 589-93.

14 Abbasi AS, Allen MW, De Christofaro D, Ungar J. Detection and estimation of the degree of mitral regurgitation by range-gated pulsed Doppler echocardiography. Circulation 1980; 61: 143-7.
15 Diebold B, Peronneau P, Blanchard D, et al. Noninvasive quantification of aortic regurgitation by Doppler echocardiography. $\mathrm{Br}$ Heart $\mathcal{f} 1$ 1983; 49: 167-73.

16 Weistein IR, Marbarger JP, Perez JE. Ultrasonic assessment of the St. Jude prosthetic valve: $M$ mode, two-dimensional, and Doppler echocardiography. Circulation 1983; 68: 897-905.

17 Holen J, Simonsen S, Froysaker T. An ultrasound Doppler technique for the noninvasive determination of the pressure gradient in the Björk-Shiley mitral valve. Circulation 1979; 59: 436-42.

18 Holen J, Hoie J, Semb B. Obstructive characteristics of Björk-Shiley, Hancock, and Lillehei-Kaster prosthetic mitral valves in the immediate postoperative period. Acta Med Scand 1978; 204: 5-10.

19 Holen J, Simonsen S, Froysaker T. Determination of pressure gradient in the Hancock mitral valve from noninvasive ultrasound Doppler data. Scand $\mathcal{f}$ Clin Lab Invest 1981 ; 41: 177-83.

20 Wilkes HS, Berger M, Gallerstein PE, Berdoff RL, Goldberg E. Left ventricular outflow obstruction after aortic valve replacement: detection with continuous wave Doppler ultrasound recording. $\mathcal{F} \mathrm{Am}$ Coll Cardiol 1983; 1: 550-3.

21 Williams GA, Labovitz AJ. Doppler hemodynamic evaluation of prosthetic (Starr Edwards and Björk Shiley) and bioprosthetic (Hancock and Carpentier Edwards) cardiac valves. Am $\mathcal{f}$ Cardiol 1985; 56: 325-32.

22 Simpson IA, Fisher J, Reece IJ, Houston AB, Hutton I, Wheatley DJ. Comparison of Doppler ultrasound velocity measurements with pressure differences across bioprosthetic valves in a pulsatile flow model. Cardiovasc Res (In press).

23 Simpson IA, Rodger JC, Tweddel AC, et al. Can Doppler echocardiography provide an haemodynamic assessment of mitral prosthetic function? $Z$ Kardiol 1985; 74 (suppl 7): (In press).

24 Hatle L, Angelsen B, Tromsdal A. Noninvasive assessment of atrioventricular pressure half-time by Doppler ultrasound. Circulation 1979; 60: 1096-104.

25 Berger M, Haimowitz A, Van Tosh A, Berdoff RL, Goldberg E. Quantitative assessment of pulmonary hypertension in patients with tricuspid regurgitation using continuous wave Doppler ultrasound. F Am Coll Cardiol 1985; 6: 359-65.

26 Sahn DJ. Real-time two-dimensional Doppler echocardiographic flow mapping. Circulation 1985; 71: 849-53. 